\title{
New Opportunities for Measurement Systems Thanks to New Trends in Space Laboratories
}

\author{
Isabelle Walther ${ }^{\star a}$, Nico de Rooij ${ }^{b}$, Bart van der Schoot ${ }^{c}$, and Augusto Cogolia
}

\begin{abstract}
Space biology is a young discipline that is strongly evolving thanks to the development of new measuring devices. The use of instruments equipped with new sensors and new technologies gives the opportunity to follow and understand better the experiments performed. Starting about three decades ago with relatively simple experiments aboard space laboratories like Spacelab, in the MIR station, as well as aboard biosatellites and sounding rockets, bioprocessing in space is becoming one of the interesting themes for the exploitation of the International Space Station, ISS. It nourishes hypotheses and speculations on possible commercial and medical applications. A few pharmaceutical companies manifested their interest in joint application research programs with universities and national and international space agencies. It is believed that low-g may contribute in two aspects to progress in this field: first, as a useful and non invasive tool to study important and still obscure biological events like signal transduction, gene expression, and cell proliferation. Second, low-g may favor the mass production of single cells by obtaining higher cell densities per unit culture volume as well as a smooth cell-cell aggregation and threedimensional organogenesis in the absence of sedimentation and shear forces. Technology of a high standard has to be used to obtain the best possible result in the restricted space of the ISS Laboratory. Often new space instrumentations can trigger earth-bound development and applications.
\end{abstract}

Keywords: Bioreactor · Microtechnology · Sensor · Space biology · Titration

\section{Introduction}

As biology experiments began to be performed in space [1], the instruments used were rather simple; most of them were vessels for the growth of cells having, in the best case, injection capacities. Soon scientists asked for better, more sophisticated instruments allowing, in addition to cell growth, the collection of information on the environment and consequently the regulation of certain parameters of the cultivation.

${ }^{*}$ Correspondence: Dr. I. Walther ${ }^{\mathrm{a}}$

Tel.: +41444451280

Fax: +4144 4451271

E-Mail: isabelle.walther@zeroglifetec.ethz.ch

aZero-g LifeTec GmbH, ETH Technopark

Technoparkstr. 1

$\mathrm{CH}-8005$ Zürich

bInstitute of Microtechnology

University of Neuchâtel

CH-2000 Neuchâtel

'Seyonic SA

Puits-Godet 12

$\mathrm{CH}-2000$ Neuchâtel
Monitoring the experiment is an important factor for its reproducibility. But not only monitoring and regulation are important, also an accurate transfer of the fluidic components (nutritive solution, activator, fixative) of the experiment is of great interest. Such instruments - the bioreactors - have been used in conventional earth-laboratories for a long time but in space the restricted volume and power as well as the safety constraints require that bioreactors have to be re-designed and specially developed for such uses [2].

The use of microtechnology for the realization of complex instruments offers a reduction in required resources (e.g. volume, mass, and power), as well as improved performance. For these reasons instruments with chemical, physical, and fluidic sensors have been developed. Almost fifteen years ago, as the first project for a space bioreactor was started, microtechnology was not so broadly applied. The first space bioreactor SBRI (Fig. 1) was developed by the Space Biology Group of the ETH Zurich in collaboration with Mecanex SA (Nyon) and the Institute of Microtechnology of Neuchâtel [3]. State-of-the-art silicon microfabricated components have been used to integrate the required functions into the limited avail- able instrument volume. The system uses a piezoelectric micropump and a silicon flow sensor for controlled delivery of nutrient solution to the $3 \mathrm{ml}$ reactor chamber. A microfabricated sensor incorporated in the reactor wall measures $\mathrm{pH}$, redox potential, and temperature of the culture. The $\mathrm{pH}$ of the cultivation was measured and regulated by means of coulometric generation of $\mathrm{OH}$ ions, a unique electrochemical method that eliminates the need for corrosive alkaline solutions.

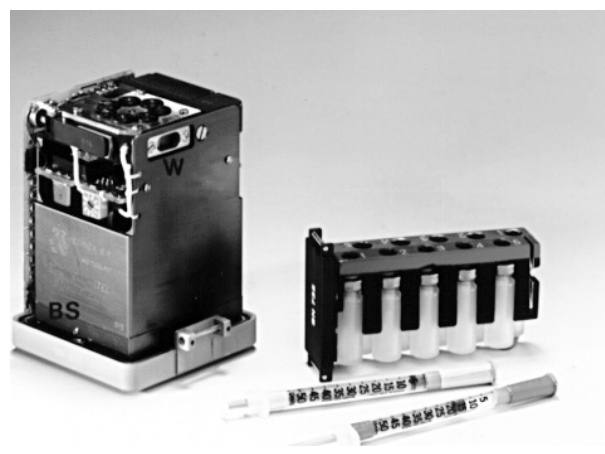

Fig. 1. SBRI with sampling bottles and syringes (W: window; BS: base for fresh and waste media storage) 
The SBRI flew in 1994 and in 1996 aboard the Columbia and Atlantis Shuttles respectively [4]. To insure a constant delivery of fresh nutrient solution during the cultivation, a flow sensor (Fig. 2) was implemented for the experiment flown in 1996 [5]. The use of these sensors for space applications opens the door to new ideas for earth-bound products. Two of them are presented in the next section: a new titration system and a fluid handling system allowing measurements in the order of the nanoliter. The latter has been developed by the spin-off company Seyonic SA. This company was created by members of the Institute of Microtechnology (Neuchâtel) based on the know-how developed during the space bioreactor project with the liquid flow sensor.

\section{Achievements}

\section{New Titration System}

The know-how developed from the bioreactor with regard to the $\mathrm{pH}$ sensor (with temperature and redox measurements) together with the coulometric generation of $\mathrm{OH}$ ions has been the basis for the development of a more complex chip integrating $\mathrm{pH}$, temperature, redox, and conductivity. This novel electrochemical titration system has been recently commercialized by Thermo (Orion Flash Titrator), which features fast titration times.

Details on the analytical performance of the Flash Titrator are described elsewhere [6]. In this paper we will focus on one adaptation in the chip technology that has made the system suitable for application in a commercial product. For this, a new technique for the alloy formation between aluminum and platinum was developed. Platinum is a widely used electrode material in electrochemistry. For many applications like conductometric and amperometric sensors a high effective surface area over the geometric surface area is desired. This is usually realized by electrodeposition of platinum-black on individual devices. For electrochemical sensors made by thin film technology, such a step is preferably performed on the whole wafer. Although possible in theory, in practice this is not as trivial as perhaps thought at first glance. All electrodes that are to be modified need to be interconnected and the exposed parts where deposition is not required have to be protected. After deposition the connections have to be cut during dicing, necessitating a proper design. Also, platinum-black is quite fragile and is easily damaged during the subsequent process steps.

To improve the alloy formation between aluminum and platinum, a method to increase the surface area has been studied. Alloy formation in thin film processing is often a subject of concern as it can have disastrous effects on the functioning of the devices.

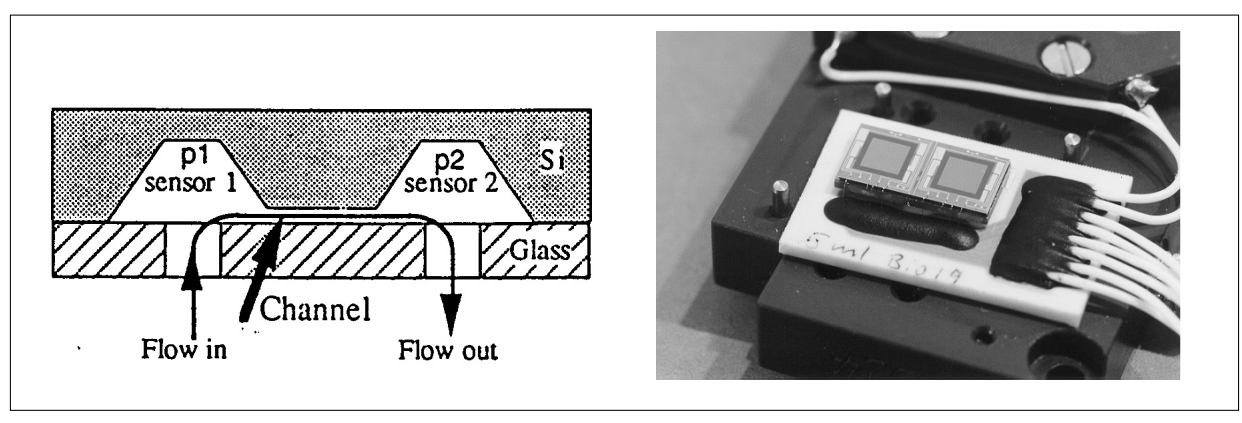

Fig. 2. Flow sensor principle and view when open

During the processing of electrochemical sensors we were also confronted with problems of alloy formation between aluminum and platinum. However, we found that when platinum had been in contact with aluminum under relatively mild conditions, the resulting electrode showed an increase of the effective surface area, while the chemical and electrochemical properties seem to stay intact. The formation of an alloy between thin film platinum and aluminum results in a material with the electrochemical properties of platinum with an increased surface roughness [7] Highest roughness factors observed were in the order of 50. Anneal temperatures above $250{ }^{\circ} \mathrm{C}$ should be avoided as this renders the electrode material resistive. The attractive feature of the proposed method is that it is fully compatible with thin film technology and the resulting material is much more robust than the normally used platinum-black. In analogy with platinum-black the term platinum-brown has been introduced. The Table gives an overview of the differences between black and brown-Pt.

Following the initial work of Bergveld et al. [8] and with these further developments, chemical sensor-actuator systems have been utilized for a number of different applications. Only recently the original ideas reappeared in a commercial product by Thermo Electron, the Orion FLASH Titrator $^{\mathrm{TM}}$ (patents applied) using a pH-ISFET chip. The success of the commercial product development can certainly be attributed to the creation of a large knowledge base and the possibility to program specific analysis methods in the instrument.

Fig. 3 shows the layout of the FLASH Titration chip with key titration features

Table. Comparison of Pt-black and Pt-brown

Pt-black

Roughness factor $>>100$

Preferably on individual devices

On-wafer homogeneity difficult

Not CMOS compatible

Fragile (needs frequent replatinisation) identified. The two critical elements are the $\mathrm{pH}$-sensitive ISFET gate and the adjacent platinum generating electrode [8]. So long as calibration is carried out, FLASH Titration does not require $100 \%$ current efficiency. And because products generated at the counter electrode do not have time to diffuse to the ISFET during the titration there is no need to provide an ion barrier such as a salt bridge or membrane as is required in a conventional coulometric titration.

\section{Precise Nanoliter Fluid Handling System}

In the space bioreactor, an accurate fluid delivery system was necessary for the correct sequence of the experiment. As the micropiezo pump is very delicate, a flow sensor was integrated after the first space flight in 1994. This sensor regulated the voltage applied to the pump in correlation with the amount of fluid passing the sensor. At that time the flow sensor was a quite new development. Based on the know-how gathered during the development of the flow-sensor, Seyonic SA was able to build a dispensing system for laboratory automation. This system for accurate low-volume delivery of liquids in the micro- to nanoliter range makes use of an integrated miniature flow sensor as part of an intelligent feedback control loop driving a micro solenoid valve [9].

The flow sensor is hydraulically connected with the pressurized system liquid in the dispensing channel and located downstream from the pressure source, above the solenoid valve. The sensor operates in a differential mode and responds in real-time to the internal flow-pulse resulting from the brief opening interval of the solenoid valve

\section{Pt-brown}

Roughness factor $40-55$

Easily done on whole wafer

Good homogeneity

$\mathrm{CMOS}^{\mathrm{a}}$ compatible

Very robust 


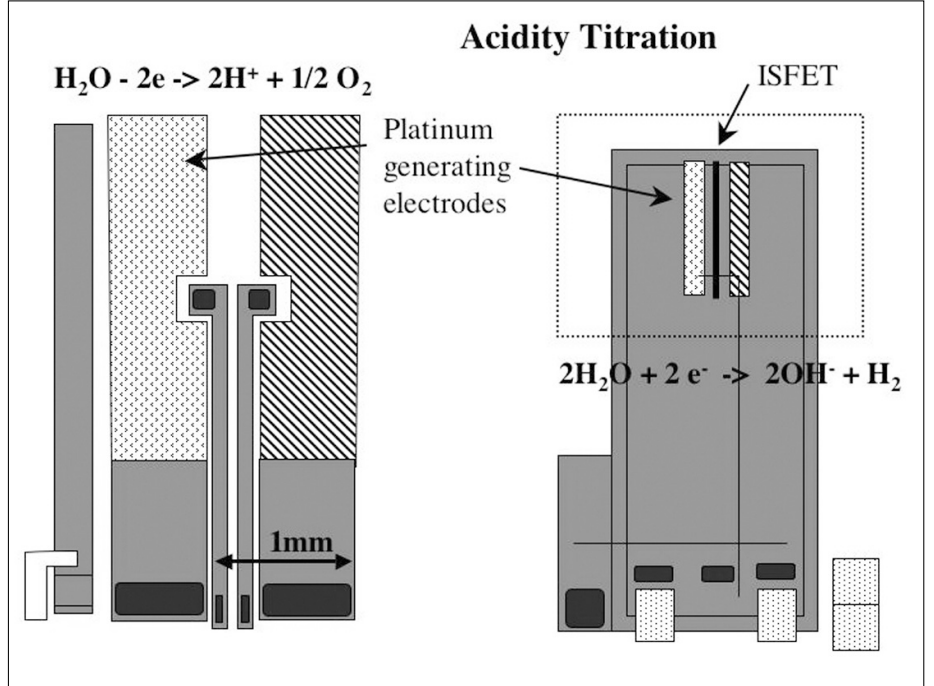

Fig. 3. FLASH Titration chip layout

leading to a rapid ejection of a fluid droplet. The integral of the flow-pulse delivered by the sensor is directly proportional to the volume of the ejected droplet from the nozzle. The quantitative information is utilized to provide active control of the effectively dispensed or aspirated volume by adjusting the solenoid valve accordingly. This process significantly enhances the precision of the fluid delivery. The system furthermore compensates automatically for any changes in the physical properties (e.g. viscosity) of the dispensed liquid. The data delivered by the flow sensor can be saved and backtracked in order to confirm and validate the aspiration- and dispense process in its entirety. The collected dispensing information can be used for quality control assessments and automatically be made part of an electronic record.

\section{The Key Element: The Flow Sensor}

The key feature of the dispenser is the differential flow sensor that acts as a 'gatekeeper' between the pressure source and the solenoid valve, in every dispensing channel.

The flow sensor consists of two piezoresistive low-pressure sensor elements for differential pressure measurement across a fluidic restriction. The pressure drop caused by viscous liquid flowing through the micromachined channel is, at low Reynolds numbers, directly proportional to the volumetric flow rate. The sensor chip is assembled in the form of a hybrid module with electronics integrated onto the ceramic substrate, providing amplified $0-5$ Volt pressure signals. It is mounted stressfree onto a ceramic substrate, with through holes for the fluidic interconnections, using silicone sealant joints and then assembled in a manifold. The dimensions of the flow sensor manifold are $9 \times 9 \times 35 \mathrm{~mm}^{3}$. Fig. 4 shows a bank of eight fully assembled dispensers consisting of the flow sensor module, solenoid valve and dispensing tip.

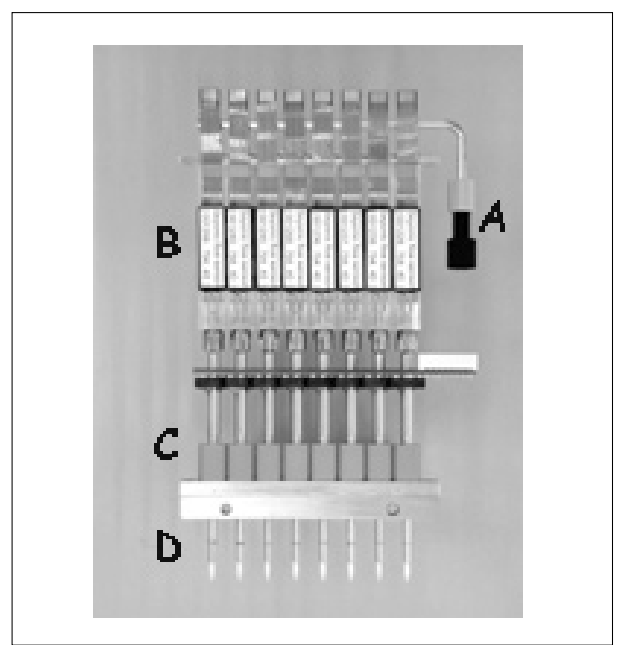

Fig. 4. Dispensing channels with inlet (A), integrated flow sensor (B), valve (C, $9 \mathrm{~mm}$ spacing), and cannula and ceramic dispensing tips (D)

\section{Conclusions - Outlook}

With the view of providing an impulse to the exploitation of space technologies for earth utilization, NASA and ESA are supporting the development of sophisticated instrumentation to be installed on board of the ISS. The ESA projects currently under study and development are the controlled environment tissue culture facility CETIC, the gradient bioreactor system GBS, and the biotechnology mammalian tissue culture facility BMTC. NASA is developing a complex apparatus called the Cell Culture Unit. The focus is on bone, cartilage, and vascular tissues with Payload System Inc. Silicon technology, sensors and liquid handling are intensively investigated.

The Space Biology Group have participated in the GBS and to the BMTC projects. The former was collaboration with Mecanex SA (Nyon) and Seyonic SA (Neuchâtel). In this instrument a gradient of activators, growth factors, and/or nutrients can be formed in the cultivation chamber and can regulate growth and differentiation of the investigated cells. A bread-board model was tested in functional tests until the end of 2004. The Space Biology Group have also been involved in the BMTC project in collaboration with Kayser-Threde (Germany) and Millenium Biologix (Switzerland).

It is still early to give a final judgment on the potential benefits of biotechnology in space. Few experiments have been carried out so far and there is only a limited database on the subject. It is also essential to complement the experiments in space with a solid program of investigations in rotating devices like clinostats, random positioning machines, and rotating wall vessels that randomize the exposure of the biological samples to the gravity vector, and thus mimic (but do not reproduce) low-gravity conditions. Nonetheless, the technical developments made for space experiments have been successfully implemented in earth-bound instruments and new companies based on the know-how gained during the space bioreactor projects have been created.

These are the first steps in a terrestrial use of space developments.

\section{Acknowledgements}

The development of the bioreactors has been supported by grants from the Prodex Program of ESA, and the ETH Zurich. The Gradient Bioreactor and BMTC studies were funded by ESA.

Received: February 8, 2005

[1] D. Moore, A. Cogoli, 'Biological and Medical Research in Space', Springer Verlag, Heidelberg. 1996, 1 .

[2] I. Walther, in 'Cell Biology and Biotechnology in Space', Ed. A. Cogoli, $A d v$. Space Biol. Med. Vol 8, Elsevier, 2000 , 197.

[3] I. Walther, R. Jeanneret, B. van der Schoot, N. de Rooij, V. Gass, C. Arquint, G. Lorenzi, B. Bechler, A. Cogoli, J. Biotechnol. 1994, 38, 21

[4] I. Walther, B. Bechler, O. Müller, E. Hunzinger, A. Cogoli, J. Biotechnol. 1996, 47, 113.

[5] I. Walther, B. van der Schoot, M. Boillat, O. Müller, A. Cogoli, Chimia 1999, 53 , 75.

[6] B. van der Schoot, P. van der Wal, N. de Rooij, S. West, Sensors and Actuators B, 2005, 105, 88 .

[7] P.D. van der Wal, M. Dadras, M. Koudelka-Hep, N.F. de Rooij, Proceedings ECSO4, 2004.

[8] P. Bergveld, B.H. van der Schoot, J.H.L. Onokiewicz, Anal. Chim. Acta 1983, 151, 143.

[9] C. Haber, M. Boillat, B. van der Schoot, ASSAY \& Drug Disc. Manuscript, 2004, 32. 